\title{
Généralisation de la similitude de M. Vantroys en tenant compte de la variation des profondeurs marines
}

\section{The generalization of Vantroys' similarity laws allowing for variations in the depth of the sea}

\author{
R. BONNEFILLE
}

INGENIEUI AU LABOHATOME NATIONAL, D'HYDRAULIQUE

\begin{abstract}
M. Vantroys a montré que la similitude aes phénomènes de marées océaniques est possible en utilisant des modeles rédnits fixes oul la force de Coriolis est négligée. Nous établissons ici les lois de similitude correspondantes, en ne négligeant pas la variation des profondeurs marines, ce qui nous conduit a introduire la notion d'échelle de similitude complexe et $\dot{a}$ préciser les conditions de détermination de la représentation conforme entre la terre et le modele.
\end{abstract}

\begin{abstract}
Vantroys showed that it is possible to simulate oceanic tides by using stationary scale models on which the Coriolis force is neglected. The associated similarity laws are fonmulated in this article and allowance is made for variations in the depth of the sea. The latter consideration maties it necessary to introduce the concept of a complex similarity scale and to define the conditions for determining conformal representation of the earth on the model.
\end{abstract}

\section{I. - INTRODUCTION}

Dans sa thèse et dans une communication présentée à la XI" Assemblée Générale de l'Association Internationale d'Océanographie Physique, M. Vantroys a montré que la similitude hydraulique des marées océaniques est possible en utilisant des modèles réduits fixes où la force de Coriolis est négligée. Plus précisément, considérons un phénomène de marée se produisant dans un océan à la surface de la terre, au sein duquel le frottement obéit à une loi linéaire avec la vitesse, et où entre en jeu pour une part importante la force de Coriolis due à la rotation de la terre; pour représenter le phénomène hydrodynamique sur modèle réduit en utilisant la similitude classique, le modèle doit être animé d'un mouvement de rotation induisant une accélération complémentaire à l'échelle. Or, M. Vantroys montre, grâce à une nouvelle théorie de similitude, que l'on peut s'affranchir de faire tourner le modèle en utilisant des échelles de similitude obéissant à de nouvelles relations. La plus caractéristique de ces nouvelles relations est une transformation linéaire à terme complexe reliant les champs de courant nature et modèle.

Ces nouvelles lois de similitude ont été établies en toute rigueur, en négligeant la variation de la profondeur de l'océan étudié. Cette théorie en première approximation n'est donc pas suffisante pour étudier sur modèle réduit fixe les marées des mers littorales, où l'on peut rencontrer des profondeurs variant de facon continue sur une grande partie du domaine maritime.

Nous présentons ici les résultats et la méthode de calcul d'une deuxième approximation de la nouvelle théorie de similitude en tenant compte de la variation des profondeurs. Nous verrons que le 
problème n'est possible que si la loi de profondeur satisfait à une équation aux dérivées partielles dépendant de la fonction de représentation conforme de la sphère terrestre sur la carte dont est issu le modèle. Nous rencontrerons aussi au cours des calculs la notion d'échelle de similitude complexe.

\section{II. - ETABLISSEMENT DES LOIS DE SIMILITUDE}

Désignons par $f(x, y)$ la fonction représentative de l'échelle de la transformation conforme de la surface terrestre sur la carte plane, par $\zeta$, $u$ et $v$ la dénivellation du plan d'eau et les composantes du courant de marée, par $\sigma$ et w la pulsation de la marée el la vitesse de rotation terrestre, par $\mathrm{L}$, $h$ et la latitude du point $x, y$ considéré, la profondeur de la mer el le coefficient de frottement linéaire. Les équations dynamiques de la marée sur la carte plane el conforme de la surface terrestre sont :

$$
\begin{array}{r}
(\hat{\imath}+i \sigma) u-\psi v=-g f \frac{\partial \zeta}{\partial x} \\
(0+i \sigma) v+\gamma u=-g f \frac{\partial \zeta}{\partial y} \\
\frac{\partial}{\partial x}(h u)+\frac{\partial}{\partial y}(h \nu)+i \sigma \frac{\zeta}{f}=0
\end{array}
$$

avec :

$$
\gamma=2 \omega \sin \mathrm{L}
$$

Dans ces relations, on suppose essentiellement que la marée est sinusoidale par rapport au temps et s'exprime par Ke $e^{i o t}$; s'il n'en était pas ainsi, on serait amené à considérer séparément chaque harmonique de la marée. On suppose, en outre, que la profondeur de la mer est grande devant l'amplitude de la dénivellation car, dans le cas contraire, la troisième équation (1), c'est-à-dire l'équation de continuité, ne peut plus s'écrire sous cette forme. Soit en effet $h_{0}$ la profondeur moyenne de lis mer; on a:

$$
h=h_{0}+\zeta
$$

$u$ et $v$ étant aussi affectés du facteur e $e^{i o t}$; le premier membre de l'équation de continuité fait apparaitre des termes en $e^{2 i s t}$ résultant des produits $\zeta u$ et $\zeta v$, ce qui entraîne l'apparition de l'harmonique 2 de $\zeta$ et ainsi de suite. On doit, si la profondeur est faible, introduire des fonctions $\zeta$, $u$ et $\nu$ développées en série de Fourier:

$$
\zeta=\sum_{n=1}^{n=\infty} \zeta_{n} e^{i n n t} \quad n=\sum_{n=1}^{n=\infty} u_{m} e^{i m n, t} \quad n=\sum_{n=1}^{n} \eta_{m} e^{i m+t}
$$

L'écuation de continuité est alors :

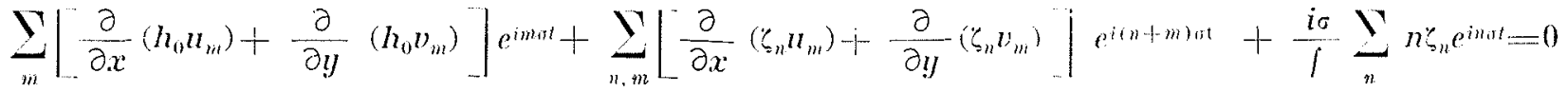

En considérant séparément le fondamental $n=m-1$ :

$$
\frac{\partial}{\partial x}\left(h_{0} u_{1}\right)+\frac{\partial}{\partial y}\left(h_{0} v_{1}\right)+\frac{i \sigma}{f} \zeta_{1}=0
$$

on retrouve l'équation de continuité du système (1) qui, jointe aux deux premières équations dynamiques (1), permet de calculer $\zeta_{1}, u_{1}$ et $v_{1}$, compte tenu des conditions aux limites. L'harmonique 2, c'est-à-dire $\zeta_{2}, u_{2}$ et $v_{2}$, est alor's déterminé à l'aide de :

$$
\frac{\partial}{\partial x}\left(h_{0} u_{2}\right)+\frac{\partial}{\partial y}\left(h_{0} v_{2}\right)+\frac{\partial}{\partial x}\left(\zeta_{1} u_{1}\right)+\frac{\partial}{\partial y}\left(\zeta_{1} v_{1}\right)+\frac{2 i \sigma}{i} \zeta_{2}=0
$$


où $u_{1}, \zeta_{1}$ et $v_{1}$ sont connus et des équations dynamiques relatives à l'harmonique 2. Ensuite on peut, par le mème procédé, calculer l'harmonique 3 , connaissant le fondamental et l'harmonique 2 , en utilisant :

$$
\frac{\partial}{\partial x}\left(h_{0} u_{3}\right)+\frac{\partial}{\partial y}\left(h_{0} v_{3}\right)+\frac{\partial}{\partial x}\left(\zeta_{1} u_{2}\right)+\frac{\partial}{\partial y}\left(\zeta_{1} v_{2}\right)+\frac{\partial}{\partial x}\left(\zeta_{2} u_{1}\right)+\frac{\partial}{\partial y}\left(\zeta_{2} v_{1}\right)+\frac{3 i \sigma}{f} \zeta_{3}=0
$$

et ainsi de suite :

Cette remarque montre que l'on peut considérer séparément l'étude de la reproduction du fondamental de la marée sur modèle réduit, bien que les profondeurs soient faibles, la déformation de la marée qui en résulte étant étudiće par ailleurs.

Posons :

$$
\left.\begin{array}{c}
\mathrm{C}=\% /(0-i \sigma) \\
a=g / \mathrm{C} / \%\left(1+\mathrm{C}^{2}\right)
\end{array}\right\}
$$

C $(x, y)$ étant une fonction complexe connuc, le systime (1) se transforme en :

$$
\begin{gathered}
u=-a\left(\frac{\partial \zeta}{\partial x}+\mathrm{C} \frac{\partial \zeta}{\partial y}\right), \quad v=-a\left(\frac{\partial \zeta}{\partial y}-\mathrm{C} \frac{\partial \zeta}{\partial x}\right) \\
\Delta \zeta+\overrightarrow{\operatorname{srad}} \zeta \overrightarrow{\mathrm{grad}} \log a h-\mathrm{C} \frac{\mathrm{D})(\zeta, \log \mathrm{C} a h)}{\mathrm{D}(x, y)}-\frac{i \sigma \zeta}{a f h}=0
\end{gathered}
$$

Construisons un modèle réduit de la carte pour lequel la période de la marée est assez petite pour que l'on puisse négliger la force de Coriolis due à la rotation terrestre. Affectons de l'indice prime les grandeurs relatives au modèle et homologues de celles de la nature. Alors $\gamma^{\prime}=0, f^{\prime}=1$, $C^{\prime}=0$ et les équations relatives au fondamental de l'onde deviennent, en confondant $h$ et $h_{0}$ :

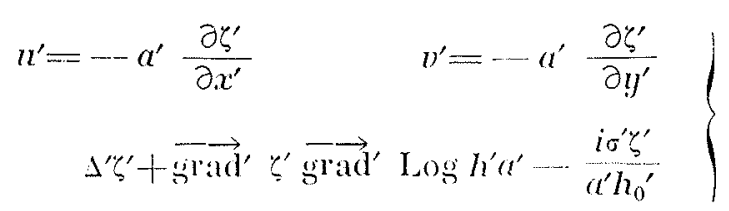

Définissons une série de rapports de similitude entre la carte plane et le modèle, ces rapports dépendant a priori des coordonnées de la carte et du modèle:

$$
\begin{array}{ll}
\lambda=d x^{\prime} / d x=d y^{\prime} / d y & : \text { échelle en plan, } \\
y=h_{0}^{\prime} / h_{0} & : \text { échelle en hauteur, } \\
\hat{\delta}=\zeta^{\prime} / \zeta & : \text { échelle des dénivellations des plans d'eau, } \\
\mathrm{T}=\sigma / \sigma^{\prime} & : \text { échelle des temps, } \\
\mathrm{R}=\rho^{\prime} / \rho & : \text { échelle des coeficients de frottement. }
\end{array}
$$

En exprimant les équations (5) en fonction des grandeurs relatives à la nature compte tenu des échelles de similitude, on obtient des équations qui doivent être identiques aux équations correspondantes (4). L'identification est possible si:

$$
\begin{aligned}
& \left.\begin{array}{l}
\frac{\partial}{\partial x} \log \frac{g^{\prime} \mathrm{T} \mu \hat{\delta}^{2}}{a(0 \mathrm{RT}+i \sigma)}=-\mathrm{C} \frac{\partial}{\partial y} \log \mathrm{C} a h_{0} \\
\frac{\partial}{\partial y} \log \frac{g^{r} \mathrm{~T} \mu \delta^{2}}{a(\bullet \mathrm{RT}+i \sigma)}=\mathrm{C} \frac{\partial}{\partial x} \log \mathrm{C} a h_{0}
\end{array}\right\} \\
& \Delta \log \partial+\overrightarrow{g r a d} \log \delta \overrightarrow{\operatorname{grad}} \log \frac{\mu \delta \hat{o} h_{0} \mathrm{~T}}{\rho \mathrm{RT}+i \sigma}=\frac{\sigma^{2}}{g h_{0} f^{2}}\left(1-\frac{\gamma^{2}}{\rho^{2}+\sigma^{2}}-\frac{\lambda^{2} f^{2}}{\mu \mathrm{T}^{2}}\right)-\frac{i \sigma \rho}{g h_{0} f^{2}}\left(1+\frac{\gamma^{2}}{\rho^{2}+\sigma^{2}}-\frac{\lambda^{2} \mathrm{R} f^{2}}{\mathrm{~T}_{\mu}}\right)
\end{aligned}
$$




$$
\begin{aligned}
& u=\frac{a \lambda\left(\rho \mathrm{R} \mathrm{T}^{\top}+i \sigma\right)}{g^{\mathrm{T}} \delta}\left(u^{\prime}+\mathrm{C} v^{\prime}\right)+\frac{a^{\prime} \zeta}{\delta}\left(\frac{\partial \delta}{\partial x}+\mathrm{C} \frac{\partial \delta}{\partial y}\right) \\
& v=\frac{a \lambda\left(\mathrm{R}^{\prime} \mathrm{T}+i \sigma\right)}{g^{\prime} \mathrm{T} \delta}\left(v^{\prime}-\mathrm{C} u{ }^{\prime}\right)+\frac{a \zeta}{\hat{\partial}}\left(\frac{\partial \hat{\partial}}{\partial y}-\mathrm{C} \frac{\partial \delta}{\partial x}\right)
\end{aligned}
$$

\section{III. - DISCUSSION DES LOIS DE SIMILITUDE}

Rappelons que les conditions de similitade (6) à (8) sont relatives à la représentation sur modele réduit non lournant du fondamental de l'onde-marée. Dans le cas d'une mer de grande profondeur, chaque harmonique de la marée conduit à un système de conditions semblables, mais avec une pulsation différente. Il n’est pas nécessaire d'étudier les harmoniques en mème temps que le fondamental, ceci en vertu de la possibilité d'addition des petits mouvements et puisque, ayant une origine extérieure au domaine étudié, ils sont indépendants du fondamental. Si les profondeurs sont faibles, les relations de similitude sont relatives au fondamental et sa reproduction est seule assurée. Il reste alors à savoir si les harmoniques d'origine interne au domaine étudié et provenant de la variation de la profondeur sont représentés à l'échelle.

La fonction C élant complexe, les égalités (6) el (7) représentent six équations entre six fonctions inconnues $\lambda, y, \delta, ' T$, $R$ et $f$. Le problème est done théoriquement possible.

Après avoir résolu le système des équations (6) ot (7), les conditions de similitude (6) représentent des relations linéaires entre le champ de courant nature $(u, v)$, le champ de courant modèle $\left(u^{\prime}, v^{\prime}\right)$ et la marée nature $\zeta$. Tous les coefficients variables avec l'espace de cette transformation sont connus. Elles constituent les échelles de similitude de courant, le mot échelle étant pris dans le sens général de l'opération de correspondance biunivoque.

Les considérations précédentes ayant montré que les échelles généralisées sont des fonctions de l'espace, il est intéressant de compléter cette généralisation en introduisant la notion d'échelle complexe. En eflet, considérons une grandeur physique fonction sinusö̉ale du temps, représentée lans les calculs par une fonction complexe de l'espace telle que $\zeta(x, y)$ par exemple. Il est possible d'appliquer à cette grandeur une échelle complexe; le module de l'échelle s'appliquera au module de la fonction complexe représentant la grandeur physique, éestà-dire à l'amplitude de cette grandeur; l'argument de l'échelle modifiera l'argument de la grandeur physique, c'est-à-dire la phase de ce phénomène.

Dans le problème qui nous préoccupe, seules les grandeurs $\zeta, u$, et $v$ sont complexes. On a vu que les échelles de courant sont une transformation complexe; il reste à définir une échelle des dénivellations ò complexe, soit :

$$
\delta=\hat{o}_{0} e^{i \varphi}
$$

Dans ces conditions, les relations de similitude (6) et (8) restent inchangées, puisque, dans l'équation de continuité, on a remplacé $h$ par $h_{0}$ pour la recherche du fondamental.

Par contre, lorsqu'on voudra étudier les harmoniques d'origine interne au domaine, un problème se posera alors, car les équations :

$$
h=h_{0}+\zeta, \quad h h^{\prime}=h_{0}^{\prime}+\zeta^{\prime}
$$

seront à prendre en considération; a priori, elles conduisent à la condition $\delta=u$. et $\delta$, comme $\mu$, ne peut ètre une fonction complexe.

L'avantage d'introduire une échelle o complexe est d'avoir une septième inconnue à déterminer par le système (6) et (7). Dans ce cas, une échelle devient arbitraire; on choisira l'échelle des temps indépendante de l'espace $\mathrm{T}=\mathrm{T}_{0}=\mathrm{C}^{\text {te }}$, car cette condition est physiquement nécessaire. En effet, dans la nature, la pulsation $\sigma$ est constante; si ' $\mathrm{T}$ était fonction de l'espace, la pulsation $\sigma$ ' serait variable avec l'espace sur le modèle, ce qui est impossible étant donné le support physique utilisé pour le modèle, c'est-à-dire la propagation d'onde au sein d'un fluide incompressible.

Remarquons alors que si, pour représenter les harmoniques, on suppose o réel, c'est-à-dire o $=0$, les six conditions (6) et (7) montrent que $h$ et $p$ doivent satisfaire à une certaine relation. 


\section{IV. - RESOLUTION DES LOIS DE SIMILITUDE}

Les relations (6) permettent d'expliciter l'équation donnant la fonction $f$ contenue dans le paramètre $a$. En effet, on obtient à partir de (3) et (6) la condition :

$$
\Delta \log \frac{g h l \mathrm{C}^{2}}{\gamma\left(1+\mathrm{C}^{2}\right)}+\overrightarrow{\operatorname{grad}} \log \mathrm{C} \cdot \overrightarrow{\operatorname{grad}} \mathrm{Log} \frac{g h f \mathrm{C}^{2}}{\gamma\left(1+\mathrm{C}^{2}\right)}=0
$$

C'est une équation aux dérivées partielles du seeond ordre en $f(x, y)$ en fonction de $g h$, p et $\mathrm{L}(x, y)$ et des constantes $\omega$ et $\sigma$, En posant:

$$
\mathrm{F}=\log \frac{g h f \mathrm{C}^{2}}{\gamma\left(1+\mathrm{C}^{2}\right)}
$$

cette équation s'écrit, compte tenu des expressions de $\gamma$ et $\mathrm{C}$ :

$$
\Delta \mathrm{F}+\operatorname{cotg} \mathrm{L} \overrightarrow{\mathrm{grad}} \mathrm{F} \overrightarrow{\operatorname{grad}} \mathrm{L}-\overrightarrow{\operatorname{grad}} \mathrm{F} \overrightarrow{\operatorname{grad}} \log (\varphi+i \sigma)=0
$$

On voit que $f$ devant être réel, dans le cas général, il faut que $h$ et $p$ satisfassent à une relation. La fonction $f$, qui représente l'échelle de la représentation de la sphère terrestre sur la carte, étant déterminée, il reste à calculer la représentation conforme correspondante et à quelles conditions le probleme est possible, ces conditions devant se traduire par une relation entre les fonetions $h$, : et l'espace $x, y$.

Si (9) est vérifié, l'expression sous le signe «somme "suivante est intégrable; on peut poser :

$$
\int \mathrm{C} \frac{\partial \mathrm{F}}{\partial y} d x-\mathrm{C} \cdot \frac{\partial \mathrm{F}}{\partial x} d y=\mathrm{A}+i \mathrm{~B}
$$

les équations (6) se réduisent alors à :

$$
\frac{\mathrm{T}_{0}{ }_{0 . \delta_{0}}^{2} e^{2 i \varphi}}{\rho \mathrm{RT}_{0}+i \sigma}=\frac{\mathrm{K} f \mathrm{C}}{\gamma\left(1+\mathrm{C}^{2}\right)} e^{-\mathrm{A}-i B}
$$

K étant une constante, cette égalité exprime $\delta_{11}, \varphi, \mu, \mathrm{R}$ en fonction de $x$ et $y$.

On peut alors éliminer $a /\left({ }_{0} \mathrm{RT}+i_{\sigma}\right)$ du premier membre de $(7)$; si on désigne par a et $\beta$ les parties réelles et imaginaires de ce premier membre, $\alpha$ et $\beta$ ne sont fonction que de $\delta_{0}$, $v$ et $x, y$. L'équation (7) se décompose en :

$$
\left.\begin{array}{l}
\lambda^{2}=\frac{\mu \mathrm{T}_{11}{ }^{2}}{f^{2}}\left(1-\frac{\gamma^{2}}{\rho^{2}+\sigma^{2}}-\frac{g h \alpha f^{2}}{\sigma^{2}}\right) \\
\mathrm{RT}_{0}=\frac{1+\left[\gamma^{2} /\left(\sigma^{2}+\sigma^{2}\right)\right]+\left(g h \beta f^{2} / \sigma \sigma\right)}{1-\left[\gamma^{2} /\left(\sigma^{2}+\sigma^{2}\right)\right]-\left(g h \alpha f^{2} / \sigma^{2}\right)}
\end{array}\right\}
$$

Les quatre équations (10) et (1.1) permettent de calculer sans intégration les inconnues $\hat{o}_{0}, \hat{\rho}$, $\mu, \quad$ R et $\lambda$. On voit que l'une d'elles est arbitraire, cette possibilité provenant de ce que la condition $f$ réel conduit à une relation entre $h$ et $q$. Les conditions de courant (8) s'écrivent alors :

$$
\begin{aligned}
& u=\frac{\sqrt{\mu}}{\delta} \frac{u^{\prime}+\frac{\gamma v^{\prime}}{\rho+i \sigma}}{\sqrt{1-\frac{\gamma^{2}}{\rho^{2}+\sigma^{2}}-\frac{g h f^{2} \alpha}{\sigma^{2}}}} \cdot \frac{p\left(1+\frac{\gamma^{2}}{\gamma^{2}+\sigma^{2}}+\frac{g h f^{2} \beta}{\sigma \rho}\right)+i \sigma\left(1+\frac{\gamma^{2}}{\rho^{2}+\sigma^{2}}-\frac{g h f^{2} \alpha}{\sigma^{2}}\right)}{\rho\left(1+\frac{\gamma^{2}}{\rho^{2}+\sigma^{2}}\right)+i \sigma\left(1-\frac{\gamma^{2}}{\rho^{2}+\sigma^{2}}\right)} \\
& +\frac{g f \zeta\left(\frac{\partial \hat{c}}{\partial x}+\frac{\gamma}{\hat{\imath}+i \sigma} \frac{\partial \delta}{\partial y}\right)}{?\left(1+\frac{\gamma^{2}}{\rho^{2}+\sigma^{2}}\right)+i \sigma\left(1-\frac{\gamma^{2}}{\rho^{2}+\sigma^{2}}\right)}
\end{aligned}
$$


et une formule analogue pour $b$, en intervertissant les grandeurs $u^{\prime}$ et $v^{\prime}$ et en changeant le signe de $\gamma$.

Si la profondeur est faible, on doit se préoccuper des harmoniques de la marće; pour cela, on supposera $\varphi=0$, de façon à ce que o soit réel. Dans les autres cas, il est intéressant de simplifier les calculs en choisissant $\delta_{0}=C^{\text {le, }}$, d'où $\alpha=\beta=0$; alors :

$$
\begin{aligned}
\lambda & =\frac{\mathrm{T}_{0}}{f} \sqrt{\mu\left(1-\frac{\gamma^{2}}{\rho^{2}+\sigma^{2}}\right)} \\
R \mathrm{~T}_{0} & =\frac{\rho^{2}+\sigma^{2}+\gamma^{2}}{\rho^{2}+\sigma^{2}-\gamma^{2}}
\end{aligned}
$$

$\mu$ et étant obtenus directement à partir de (10); ensuite :

$$
\begin{aligned}
& u=\frac{\sqrt{\mu}}{\delta_{0} \sqrt{1-\left[\gamma^{2} /\left(\rho^{2}+\sigma^{2}\right)\right]}}\left(u^{\prime}+\frac{\gamma v^{\prime}}{p+i \sigma}\right) \\
& v=\frac{\sqrt{\mu}}{\sqrt{1-\left[\gamma^{2} /\left(p^{2}+\sigma^{2}\right)\right]}}\left(v^{\prime}-\frac{\gamma u^{\prime}}{\rho+i \sigma}\right)
\end{aligned}
$$

Dans le cas général, le degré de liberté des équations (10) et (11) peut ètre utilisé pour obtenir des échelles positives et réelles, cctte éventualité n’étant pas évidente quand on considère la première relation (11).

\section{V. - CAS PARTICULIERS}

1" Frottementr nut $=0$; c'est le cas des marées océaniques; $f$ est réel quel que soit $h$; $\mathrm{C}=-i \% / \sigma, \mathrm{A}=\mathbf{0}$, on choisit $\delta_{0}$ constante; il vient d'après (10) et (11), $\mu_{0}$ et $o_{0}$ étant des constantes :

$$
\mu=\mu_{0} f \sigma^{2} /\left(\sigma^{2}-\gamma^{2}\right), \quad \varphi=\varphi_{0}-(\mathrm{B} / 2), \quad \lambda=\frac{\mathrm{T}_{0} \sqrt{\mu_{0}}}{\sqrt{f}}
$$

$2^{\circ}$ Mer peu Étendue. - On peut supposer $g$ et $\gamma$ constants sur loute l'étendue du domaine étudie, qui n'occupe qu'une petite portion de la surface terrestre. Un cas intéressant est celui où $=C^{\text {te: }}$ l'équation (9) se réduit alors à :

Posons :

$$
\Delta \log h f=0
$$

$$
\mathrm{I}=\frac{\gamma^{\prime}}{\rho^{2}+\sigma^{2}} \int \frac{1}{h f}\left(\frac{\partial h f}{\partial y} d x-\frac{\partial h f}{\partial x} d y\right)
$$

il vient :

$$
\begin{gathered}
\mathrm{A}=\rho \mathrm{I} \quad \mathrm{B}=-\sigma \mathrm{I} \\
\left.\delta_{0}=\mathrm{C} \mathrm{tc}, \quad \mu=\mu_{0} e^{-\rho \mathrm{I}}, \quad \lambda=\frac{\lambda_{0}}{f} e^{-\beta \mathrm{I} / 2}, \vartheta=\varphi_{0}\right)+\sigma \mathrm{I} / 2, \quad \mathrm{R}=\mathrm{R}_{0}=\frac{\rho^{2}+\sigma^{2}+\gamma^{2}}{\mathrm{~T}_{0}\left(\rho^{2}+\sigma^{2}-\gamma^{2}\right)}=\mathrm{C}^{\mathrm{te}}
\end{gathered}
$$

avec:

$$
\lambda_{0}=\mathrm{T}_{0} \sqrt{\mu_{0}\left(1--\frac{\gamma^{2}}{\rho^{2}+\sigma^{2}}\right)}=\mathrm{C}^{\mathrm{te}}
$$

$3^{\circ}$ Mer peu Étendul a profondeur er frottrement constants. - En prenant $f=1$, il vient $\mathrm{I}=0$, d'où :

$$
\begin{gathered}
\delta_{0}=\mathrm{C}^{\mathrm{t},}, \quad \mu_{0}=!_{0}, \quad \lambda=\mathrm{T}_{0} \sqrt{\mu_{0}\left(1-\frac{\gamma^{2}}{\rho^{2}+\sigma^{2}}\right)} \\
\mathrm{R}_{0}=\frac{1}{\mathrm{~T}_{0}} \frac{\rho^{2}+\sigma^{2}+\gamma^{2}}{\rho^{2}+\sigma^{2}-\gamma^{2}}
\end{gathered}
$$


Toutes les échelles sont constantes; seule l'échelle des courants est une transformation linéaire de la forme :

$$
\begin{aligned}
& u=\frac{\mu_{0} \mathrm{~T}_{0}}{\partial_{0} \lambda}\left(u^{\prime}+\frac{\gamma v^{\prime}}{i+i \sigma}\right) \\
& b=\frac{p_{010} \mathrm{~T}_{0}}{\hat{\partial}_{0} \lambda}\left(v^{\prime}-\frac{\gamma u^{\prime}}{\gamma+i \sigma}\right)
\end{aligned}
$$

On retrouve les échelles de similitudes déjà proposées par M. Vantroys.

\section{VI. - REPRESENTATION CONFORME DU GLOBE TERRESTRE SUR LA CARTE}

Les coordonnées d'un point sont, à la surface de la terre, la latitude $\mathrm{L}[-(\pi / 2)<\mathrm{L}<(\pi / 2)]$ et la longitude $M(-\pi<\mathrm{M}<\pi)$, et sur la carte $x$ et $y$. La représentation conforme de la sphère terrestre sur la carte se traduit par deux fonctions:

$$
x=\mathrm{X}(\mathrm{L}, \mathrm{M}), \quad y=\mathrm{Y}(\mathrm{L}, \mathrm{M})
$$

Le rayon de la terre supposé sphérique étant pris comme unité de longueur, l'élément d'arc sur le globe terrestre est :

et sur la carte :

$$
d \mathrm{~S}=\left(d \mathrm{~L}^{2}+d \mathrm{~N}^{2} \cos ^{2} \mathrm{~L}\right)
$$

$$
d s=f d S=\left(d x^{2}+d y^{2}\right) !
$$

Lat transformation entre $d s$ et $d S$ est conforme si :

$$
\frac{\partial \mathrm{X}}{\partial \mathrm{L}} \cos \mathrm{I}=\frac{\partial \mathrm{Y}}{\partial \mathrm{M}}, \quad \frac{\partial \mathrm{X}}{\partial \mathrm{M}}=-\frac{\partial \mathrm{Y}}{\partial \mathrm{L}} \cos \mathrm{L}
$$

Les équations sont simplifiées si on remplace la coordonnée $L$ par $P$ définie par :

$$
\sin \mathrm{L}=\text { th } \mathrm{P}
$$

Les équations aux dérivées partielles définissant $\mathrm{X}$ et $\mathrm{Y}(\mathrm{P}, \mathrm{M})$ sont alors :

$$
\Delta \mathrm{X}=\Delta \mathrm{Y}=0
$$

L'expression de $f$ en fonction de X, Y, P, M est :

$$
f=\operatorname{ch} \mathrm{P} \sqrt{\left(\frac{\partial \mathrm{X}}{\partial \mathrm{P}}\right)^{2}+\left(\frac{\partial \mathrm{X}}{\partial \mathrm{M}}\right)^{2}}=\operatorname{ch} \mathrm{P} \sqrt{\left(\frac{\partial \mathrm{Y}}{\partial \mathrm{P}}\right)^{2}+\left(\frac{\partial \mathrm{Y}}{\partial \mathrm{M}}\right)^{2}}=\operatorname{ch~P} \sqrt{\frac{\mathrm{D}(\mathrm{X}, \mathrm{Y})}{\mathrm{D}(\mathrm{P}, \mathrm{M})}}
$$

Avec les nouvelles coordonnées P et M, l'équation (9) devient :

$$
\Delta \mathrm{F}+\frac{1}{\operatorname{sh} \mathrm{Pch} P} \frac{\partial \mathrm{F}}{\partial \mathrm{P}}-\overrightarrow{\operatorname{grad}} \mathrm{F} \overrightarrow{\operatorname{grad}} \log (\varphi+i \sigma)=0
$$

Posons :

$$
\mathrm{H}(\mathrm{P}, \mathrm{M})=\log \frac{2 \omega g h \operatorname{sh} \mathrm{P}}{(\rho+i \sigma)^{2}+4 \omega^{2}+h^{2} \mathrm{P}}
$$

Compte tenu des expressions de $\mathrm{F}$ et $f$, (9) est remplacée par :

$$
\begin{aligned}
& {\left[\operatorname{sh} P \operatorname{ch} P\left(\Delta H-\frac{\overrightarrow{g r a d} H \operatorname{grad} ?}{q+i \sigma}\right)\right]\left[\left(\frac{\partial X}{\partial P}\right)^{2}+\left(\frac{\partial X}{\partial M}\right)^{2}\right]}
\end{aligned}
$$

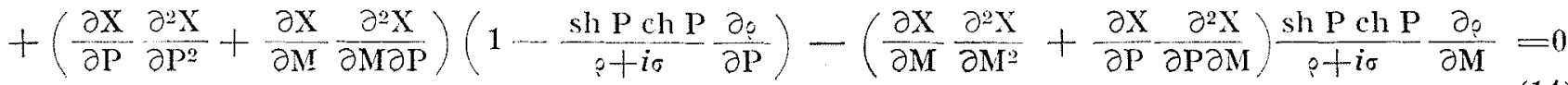


La solution du problème consiste à choisir une fonction harmonique $\mathrm{X}(\mathrm{P}, \mathrm{M})$ satisfaisant à (14). Il n'est pas évident a priori que le problème soit toujours possible, quelles que soient les fonctions $h(\mathrm{P}, \mathrm{M})$ et $(\mathrm{P}, \mathrm{M})$. Il est donc possible, dans le cas général, que $h$ et p doivent satisfaire à une iquation aux dérivées partielles dépendant éventuellement du choix de la transformation conforme de la sphère terrestre sur la carte du modèle.

Examinons le cas du frottement mul; l'équation (14) devient: $\left.\operatorname{sh} \mathrm{P} \operatorname{ch} \mathrm{P} \Delta \log g h+\frac{\partial}{\partial \mathrm{P}} \log g h+\frac{16 \omega^{2} \text { th } \mathrm{P}}{\left(\operatorname{ch}^{2} \mathrm{P}\left(\sigma^{2}-4 \omega^{2} \operatorname{th}^{2} \mathrm{P}\right)^{2}\right.}\left[\sigma^{2}\left(1-\operatorname{sh}^{2} \mathrm{P}\right)+4 \omega^{2} \operatorname{th}^{2} \mathrm{P} \operatorname{sh}^{2} \mathrm{P}\right)\right]$

$$
+\frac{(\partial \mathrm{X} / \partial \mathrm{P})\left(\partial^{2} \mathrm{X} / \partial \mathrm{P}^{2}\right)+(\partial \mathrm{X} / \partial \mathrm{M})\left(\partial^{2} \mathrm{X} / \partial \mathrm{M} \partial \mathrm{P}\right)}{(\partial \mathrm{X} / \partial \mathrm{P})^{2}+(\partial \mathrm{X} / \partial \mathrm{M})^{2}}=0
$$

compte tenu de ce que $\mathrm{X}$ est harmonique, on doit avoir :

$$
\begin{aligned}
\operatorname{sh} P \operatorname{ch~} \mathrm{P}(4+\Delta) \Delta & \log g h+\left[2\left(\operatorname{sh}^{2} \mathrm{P}+\operatorname{ch}^{2} \mathrm{P}\right)+1\right] \frac{\partial}{\partial \mathrm{P}} \Delta \log g h \\
+ & \frac{256 \omega^{2} \operatorname{th} \mathrm{P}}{\operatorname{ch}^{6} \mathrm{P}\left(\sigma^{2}-4 \omega^{2} \operatorname{th}^{2} \mathrm{P}\right)^{4}}\left[\sigma^{6} \operatorname{ch}^{2} \mathrm{P}+\sigma^{4} \omega^{2}\left(6-16 \operatorname{sh}^{2} \mathrm{P}+2 \operatorname{sh}^{4} \mathrm{P}-\operatorname{th}^{2} \mathrm{P}\right)\right. \\
& \left.\left.-4 \sigma^{2} \omega\right)^{4} \operatorname{th}^{2} \mathrm{P}\left(9-11 \operatorname{sh}^{2} \mathrm{P}+4 \operatorname{sh}^{4} \mathrm{P}-4 \mathrm{th}^{2} \mathrm{P}\right)+16 \omega^{6} \operatorname{th}^{4} \mathrm{P}\left(3+\operatorname{sh}^{2} \mathrm{P}+2 \operatorname{sh}^{4} \mathrm{P}\right)\right]=0
\end{aligned}
$$

c'est-à-dire que $g h$ doit satisfaire à une équation aux dérivées partielles du quatrième ordre.

\section{VII. - CONCLUSION:}

Cette étude encore incomplète a pour but de donner les bases d'une nouvelle approximation dans l'établissement des lois de similitude généralisée utilisables pour la construction des modèles réduits non tournants destinés à l'étude de la propagation de la marée, en tenant compte à la fois de la rotation terrestre et d'un frottement linéaire en fonction de la vitesse. Pour que le problème soit possible, la loi de profondeur doit vérifier une équation aux dérivées partielles ce qui, a priori, limite la généralité de l'emploi de cette méthode. De nombreuses questions soulevées au cours de cette étude restent à résoudre, telles que la reproduction des harmoniques de la marée d'origine locale. Enfin, étant donné la généralité du problème, il semble qu'il serait utile d'examiner le cas d'une représentation non conforme entre la sphère terrestre et le modèle, et aussi le cas du frottement quadratique.

\section{I S C USSION}

Président : M. Bruvel

M. le Président remercie M. Bonverrute de son exposé et de la contribution qu'il a ainsi apportée an problème de l'étude des marées sur modèle réduit.

M. Chapovthier demande si l'on a déjà essayé d'étudier les dispositions technologiques pratiques entraînées par la similitude de M. Vantroys.

M. Bonnefrlise répond que le problème a été envisagé et s'est révélé réalisable. Dans le cas d'un modèle océanique représcntant l'ensemble des mers du globe terrestre (theoric amorcée par M. Vantroys), en plus du problème de Ia réalisation des courants aux limites avec un champ complexe, il faudrait ajouter celui de l'introduction du potentiel générateur de la marée.

M. Bonnefille explique ensuite que dans cette théorie on tient compte de la variation de la latitude. La latitude réagit sur les conditions aux limites et sur l'échelle : on peut avoir $1 / 1000^{\circ}$ à l'équateur, par exemple.
M. RĔMÉNñRAs aimerait saroir comment se présente le genre de modèle proposé par M. Bonkefrles, par rapport aux modeles construits aux Etats-Unis, notamment par Von Arx pour représenter les problèmes océaniques.

D'après M. Bonnefilre, les Américains n'avaient pas connaissance de cette théorie à l'époque, et d'autre part, leurs modèles représentaient une assez grande partie de la terre, alor's que ceux considérés ici représentent une petite partie de la terre.

M. LACOMB fait remarquer que les modèles américains reproduisent ici un hémisphère, et la variation du paramètre de Coriolis avec la latitude est approximativement figurée par une augmentation de la profondeur du modèle en fonction de la latitude, la profondeur réelle des océans étant supposée constante.

M. Bonnefille précise qu'il s'agit de la théoric de 
Von Arx qui compense la variation de latitude par la variation de profondeur.

M. Bonneflute indique d'autre part, sur la demande de M. Chapouthrer, que les modèles en question sont paraboloüdes, à profondeur constante, sauf celui de Von Alx, qui est modifié de facon à tenir compte de la variation du terme de Coriolis avec la latitude. C'est un aspect de la dynamique du problème des tourbillons qui n’a pas été démontré.

\section{*}

M. Schoemaker, représentant M. Thissse, prend ensuite la parole :

« Avant tout, je tiens à vous remercier de pouvoir rapporter ici les essais que nous faisons actuellement a Delft concernant la force de Coriolis.

« Pour beaucoup de modèles réduits, il est impossible d'employer une plaque tournante et pourtant on peut connaitre l'effet de l'accelération de Coriolis.

« Nous faisons actuellement à Delft des essais pour reprodruire cet effet artificiellement dans un modèle stationnaire.

« Pour ceci, nous utilisons l'effet de Magnus : un cylindre toumant dans un courant subit une force perpendiculaire à la direction du courant. Ce cylindre produit aussi une force sur le courant qui est, selon la théorice directement proportionnelle à la vitesse du courant et à la vitesse de rotation du cylindre.

* En plaçant un certain nombre de ces cylindres dans un champ de courant, on peut reproduire l'effet de Coviolis.

« Au lieu d'exercer une force uniformément répartie, comme cela se produit dans le cas d'une plaque tournante, on fait agir cette force en un certain nombre de points de concentration.

« La thérie est simple, mais, dans la pratique, certains phénomènes secondaires compliquent les rapports théoriques,

« Nous avons réussi à construire des cylindres d'une forme spéciale qui reproduisent les relations théoriques dans une variation considérable de vitesse de courant, la rotation restant constante.

« Il y a notamment des limitations pratiques en ce qui concerne le nombre des cylindres par unité de superficie. Nous avons réussi à faire des cylindres qui produisent une force suffisamment grande, de sorte que leur nombre reste restreint.

«Des essais speciaux nous ont appris la distance maximum des cylindres, da point de vue des irrégularités de la surface de l'eau, provenant du fait que les forces agissent concentrées dans les points de concentration.

« Pour fixer les idées, je vous donne quelques chiffres : la méthode doit être appliquée dans un modèle aux échelles :

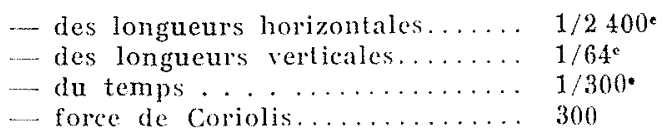

\&es cylindres ont un diametre de $2 \mathrm{~cm}$ et sont munis d'un certain nombre de collets.

« La vitesse de rotation est $750 \mathrm{tr} / \mathrm{mm}$. La distance entre chaque cylindre est $0,7 \mathrm{~m}$.

"L'effet est purement linéaire pour les courants dans lintervalle de 0,3 à $0,05 \mathrm{~m} / \mathrm{s}$, ce qui correspond à des vitesses dans la nature de 2,4 à $0,4 \mathrm{~m} / \mathrm{s}$. En dessous de cet intervalle, l'effet diminue graduellement.

«Les essais de contróle ont été faits avec un eylindre suspendu à une balance, de telle manière que nous pouvons mesurer les forces et les couples en variant les vilesses de rotation.

M. le Président remercie M. Scuomaken et demande si quelqu'un a des questions à poser sur son intervention.

M. Chapouther, très intéressé par l'exposé de M. SchorMaker, demande

1" Si l'on peut concilier les conditions d'une action aussi infinitésimale que possible, et d'une non-perturbation de l'écoulement;

2. Si la distance entre deux eylindres est suffisante pour que l'on ait un écoulement qui reproduise celui d'un modèle toumant

3 "Quel est le modèle des cylindres.

M. Schomaker explique que l'influence mutuelle des cylindres est assez faible et que, d'autre part, on agit sur le champ total du courant.

Les dimensions des cylindres sont négligeables par rapport aux distances mutuelles, de sorte que les perlurbations ne sont pas nuisibles.

Le diamètre des cylindres est de $2 \mathrm{~cm}$ et n'a aucume rolation directe avec la distance entre chacue cylindre : c'est seulement le nombre de tours/minute qui a une influence.

M. Butsel demande si le Laboratoire de Delft a pu mesurer l'effet de rugosité ou de résistance dê au dispositif proposé. On pourrait craindre, en effet, que la turbulence induite par la rotation des cylindres reste sensiblement constante, et par conséquent, que la contribution au frottement ne soit pas quadratique.

M. Schonmarer précise que la résistance introduite par la rotation des eylindres est petite par rapport à la résistance à introduire dans le modèle.

M. RÉménéras pense que lon pourrait remplacer les cylindres à effet Magnus par des dispositifs électromagnétiques (à champ tournant, par exemple), créant des petits tourbillons à axe vertical.

M. le Président remercie les personnes qui ont pris la parole, et les auditeurs, de l'attention qu'ils ont apportée aux questions traitées. 